\title{
Traditional uses of American plant species from the 1st edition of Brazilian Official Pharmacopoeia
}

\author{
Maria G. L. Brandão, ${ }^{*, 1,2}$ Gustavo P. Cosenza, ${ }^{1,2}$ Cristiane F. F. Grael, ${ }^{3}$ \\ Nilton L. Netto Junior, ${ }^{4}$ Roberto L. M. Monte-Mór ${ }^{5}$ \\ ${ }^{1}$ DATAPLAMT - Museu de História Natural e Jardim Botânico, Universidade Federal de Minas Gerais, Rua \\ Gustavo da Silveira, 1035, 30180-010 Belo Horizonte-MG, Brazil, \\ ${ }^{2}$ Laboratório de Farmacognosia, Faculdade de Farmácia, Universidade Federal de Minas Gerais, Av. Antônio \\ Carlos, 6627, 31270-901 Belo Horizonte-MG, Brazil, \\ ${ }^{3}$ Depto de Farmácia, Universidade Federal dos Vales do Jequitinhonha e Mucuri, Campus Universitário, \\ 39100-000 Diamantina-MG, Brazil, \\ ${ }^{4}$ Curso de Farmácia, Centro Universitário UNIEURO, Av. das Nações, Trecho 0, Conjunto 5 , \\ Asa Sul, 70390-130 Brasilia-DF, Brasil, \\ ${ }^{5}$ CEDEPLAR, Faculdade de Ciência Econômicas, Universidade Federal de Minas Gerais, Av. Antônio Carlos, \\ 6627, 31270-901 Belo Horizonte-MG, Brazil
}

\begin{abstract}
RESUMO: "Usos tradicionais das plantas Americanas presentes na 1ª Edição da Farmacopéia Brasileira". A $1^{\text {a }}$ Edição da Farmacopéia Brasileira, publicada em 1929, é uma rica fonte de informação sobre as plantas medicinais nativas das Américas, uma vez que ela lista as espécies usadas na medicina tradicional e oficial da época. Neste estudo, foi feita uma extensa revisão sobre os usos tradicionais de plantas descritas em oitenta e sete Monografias presentes na FBRAS $1^{\text {a }}$ Edição, em vinte livros publicados do século 19 até a década de 1970. Oitenta e seis diferentes usos medicinais foram citados e três ou mais livros; mesmos usos foram descritos em dez ou mais referências, mostrando seu amplo emprego e importância como medicamento. Os resultados mostram que as espécies nativas presentes na $1^{\text {a }}$ Edição da FBRAS têm longa tradição em uso, confirmado pelos dados históricos. É necessário, portanto, que estudos farmacológicos com essas plantas sejam estimulados, como forma de promover seu melhor aproveitamento.
\end{abstract}

Unitermos: Plantas nativas da América, Farmacopéia, usos tradicionais.

\begin{abstract}
The first edition of the Brazilian Official Pharmacopoeia (FBRAS), published in 1929, is a rich source of information about American medicinal plants, since it lists species used in both traditional and conventional medicine. In this study, we have performed a survey of the traditional uses of plants described in eighty-seven Monographs from the FBRAS in twenty bibliographies written from the $19^{\text {th }}$ century to the 1970 s. Eighty-six different traditional uses are described in three or more books; some of them were cited in ten or more books, illustrating their widespread use and importance in medicine. The species from the first edition of the FBRAS have a long tradition of medical utility, which is confirmed by historical records. In surveying these medically relevant species, we hope to encourage policy makers and the scientific public as a whole to engage in a strong debate in an attempt to improve and facilitate the pharmacological study of these species.
\end{abstract}

Keywords: American native plants, Pharmacopoeia, traditional uses.

\section{INTRODUCTION}

Latin-American countries are rich in medicinal species due to the vast plant biodiversity and a rich tradition of plant utilization practiced by Americans for centuries. In Brazil, however, the intense mixture of cultures (Native, African and European) led to an introduction of species native from other continents. The accelerating destruction of Brazil's botanically rich native ecosystems is also contributing to a gradual loss of knowledge about the native plants used in traditional medicine (Dean, 1995; Shanley and Rosa, 2005; Brandão and Montemor, 2008). Native medicinal species were also used for decades by pharmaceutical companies in Brazil for preparing commercial products. The lack of pharmacological studies on the efficacy and toxicity of these species, however, is promoting a progressive substitution of them by species from other continents in the manufacture of these products (Brandão et al., 2009; Carvalho et al., 2008). This situation illustrates the growing need to recuperate data about native American plant species and to promote studies on them.

Information on the use of native American species in Brazil can be obtained in older bibliographies. 
Travel diaries written by European naturalists that traveled throughout Brazil in the 19th century, for example, are rich in descriptions of the use of native medicinal plants by the Brazilians (Brandão et al., 2008a). The first edition of the Brazilian Official Pharmacopoeia (FBRAS), published in 1929, is also a very important document, since it listed plant species used in the practice of both traditional and conventional medicine. In previous studies, we listed the plants and botanical products described in this edition of the FBRAS, and discussed the trend towards intense replacement of native species by others products in recent decades (Brandão et al., 2006; 2008b). In the present study, we show the results of an extensive revision of the traditional uses of American plant species from the first edition of the FBRAS in twenty books and other bibliographies about the use of plants written in Brazil from $19^{\text {th }}$ century to the 1970 s decade.

\section{MATERIAL AND METHODS}

\section{Ethnopharmacological survey on books of the $19^{\text {th }}$ century}

Six books were surveyed about the use of medicinal plants in the $19^{\text {th }}$ century (Table 1 ). The oldest were written by the French A. Saint-Hilaire (17791853) and the German K.F. von Martius (1794-1868), both naturalists who traveled throughout Brazil in the $19^{\text {th }}$ century (Brandão et al., 2008b). The contributions they made to knowledge of the Brazilian flora are incalculable - thousands of new plants were discovered and innumerable new genera were described, based on the material they collected. The use of some native species were detailed by Saint-Hilaire in his book Plantes Usuelles des Brésilien (Saint-Hilaire, 1824) and Florae Brasilae Meridionalis (1825-1852) as did von Martius in Systema de Materia Medica Vegetal (Martius, 1843). Two other influential books from the $19^{\text {th }}$ century were written by the botanists J.M. Caminhoá (1836-1896) and Theodor Peckolt (1822-1912). Caminhoá, who was influential in the $19^{\text {th }}$ century Brazilian empire, wrote a vast bibliography about medical materials, including the Elementos de Botânica Geral e Médica (1877). T. Peckolt was a German botanist who came to work in Brazil in 1847, and revised the material collected by K.F.P. Martius. He wrote extensive medical literature, with almost 170 publications, including the series of books used in this study entitled Historia das Plantas Medicinaes e Úteis do Brazil, which were published between 1887 and 1896 (Hering, 1912). Another 19 ${ }^{\text {th }}$ century author was Piotr Czerniewicz, known as Pedro Luiz Napoleão Chernoviz (1812-1881), who left Poland in 1840 to live in Rio de Janeiro. In 1841, Chernoviz published the Formulário e Guia Medico, widely used by the population in areas bereft of doctors. In the $8^{\text {th }}$ Edition (1868), he included the descriptions and uses of almost 200 native Brazilian plant species. This book remained widely used as a medical manual by official medical institutions in Brazil, until it was finally replaced

Table 1. Year of publication, author and title of the books used in the study.

\begin{tabular}{|c|c|c|c|}
\hline $\begin{array}{l}\mathrm{N}^{\circ} \text { in } \\
\text { Table } 2\end{array}$ & $\begin{array}{l}\text { Year of } \\
\text { publication }\end{array}$ & Author & Title of the book \\
\hline (1) & 1824 & A. Saint-Hilaire & Plantes Usuelles des Brésiliens. \\
\hline (1a) & $1825-1852$ & A. Saint-Hilaire & Flora Brasiliae Meridionalis \\
\hline (2) & 1843 & K.F. Von Martius & Systema de Materia Medica Vegetal Brasileira \\
\hline (3) & 1877 & J.M. Caminhoá & Elementos de Botânica Geral e Médica. \\
\hline (4) & $1887-1896$ & Peckolt and Peckolt & Historia das Plantas Medicinaes e Uteis do Brazil. \\
\hline$(5)$ & 1911 & J. Monteiro da Silva & Contribuição para o estudo da Flora Brazileira \\
\hline (6) & $1926-1978$ & M.P. Corrêa & Dicionário das Plantas Úteis do Brasil e das Exóticas Cultivadas. \\
\hline (7) & 1930 & Araújo \& Lucas & Catálogo de Extratos Fluidos. \\
\hline$(8)$ & 1939 & F.C. Hoehne & Plantas e Substâncias Vegetais Tóxicas e Medicinais. \\
\hline (9) & 1940 & J. Badini & Arquiclamídeas Medicinais de Ouro Preto. \\
\hline (10) & 1941 & M. Penna & Dicionário Brasileiro de Plantas Medicinais. \\
\hline (11) & 1942 & R. Coimbra & Notas de Fitoterapia. \\
\hline$(12)$ & 1947 & P. Le Cointe & Amazônia Brasileira. Árvores e Plantas Úteis. \\
\hline (13) & 1954 & $\begin{array}{l}\text { Laboratórios } \\
\text { Catedral }\end{array}$ & $\begin{array}{l}\text { Dicionário de Sinônimos das Plantas Medicinais } \\
\text { Brasileiras Industrializadas pelo Laboratório Catedral. }\end{array}$ \\
\hline$(14)$ & 1960 & R. Braga & Plantas do Nordeste, especialmente do Ceará. \\
\hline$(15)$ & 1965 & G.L. Cruz & Livro Verde das Plantas Medicinais e Industriais do Brasil. \\
\hline$(16)$ & 1967 & A. Balbach & As Plantas Curam. \\
\hline$(17)$ & 1996 & P.L.N. Chernoviz & Formulário e Guia Médico* \\
\hline$(18)$ & 1997 & F.J.A. Matos & O Formulário Fitoterápico do Professor Dias da Rocha* \\
\hline (19) & 1999 & Botsaris \& Machado & Flora Medicinal: Memento Terapêutico* \\
\hline
\end{tabular}

* more recent editions/ publications but with informations from 1860 to 1930. 
Table 2. Traditional uses of Brazilian plants from the first edition of the FBRAS.

\begin{tabular}{|c|c|}
\hline $\begin{array}{l}\text { Name of Monography/ part } \\
\text { Species/ FAMILY }\end{array}$ & Uses in books of the 19th century until the $1970 \mathrm{~s}$ \\
\hline $\begin{array}{l}\text { Abacateiro/ leaves } \\
\text { Persea gratissima L/ Lauraceae }\end{array}$ & 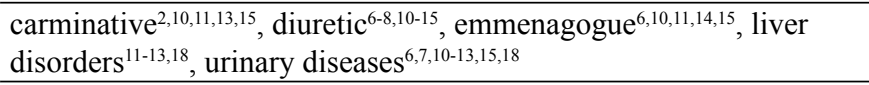 \\
\hline $\begin{array}{l}\text { Abútua/ roots } \\
\text { Chondrodendron platiphyllum (A.St-Hil.) Miers; } \\
\text { Cocculus platyphyllum; Cissampelos sp./ } \\
\text { MENISPERMACEAE }\end{array}$ & 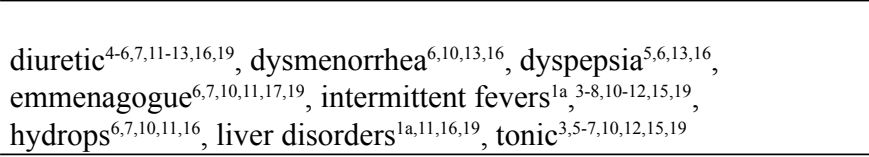 \\
\hline $\begin{array}{l}\text { Agoniada/ rind } \\
\text { Plumeria lancifolia Müll. Arg./ P. phagedaenica Mart./ } \\
\text { APOCYNACEAE }\end{array}$ & 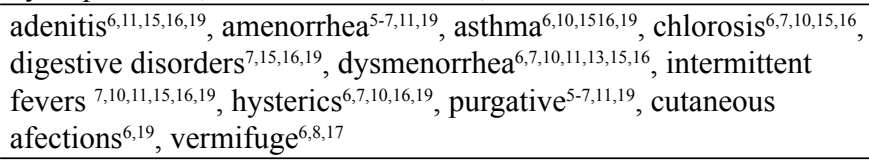 \\
\hline $\begin{array}{l}\text { Agrião do Pará/ leaves and flowers } \\
\text { Spilanthes acmella (L.) Murray; S. oleraceae L./ } \\
\text { ASTERACEAE }\end{array}$ & $\begin{array}{l}\text { anti-scorbutus }{ }^{7,12,15-17}, \text { anemia } \\
6,7,15,16,15,17, \text { odontalgia }^{6-8,12,15-17}\end{array}$ \\
\hline $\begin{array}{l}\text { Alecrim bravo/ flowers } \\
\text { Hypericum laxiusculum A. Saint-Hil./ HyPERICACEAE }\end{array}$ & anti-spasmodic ${ }^{6,8,10}$, snake bites ${ }^{1 \mathrm{la}, 2,6,8,10,17}$, vulnerary ${ }^{2,6,8,10,17}$ \\
\hline $\begin{array}{l}\text { Algodoeiro/ rind of roots } \\
\text { Gossypium herbaceum L./ MALVACEAE }\end{array}$ & 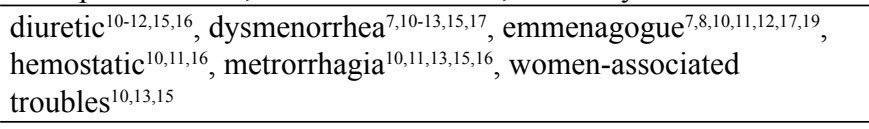 \\
\hline $\begin{array}{l}\text { Angico/ rind } \\
\text { Piptadenia colubrina (Vell.) Benth./ Anadenanthera } \\
\text { colubrina (Vell.) Benth./ FABACEAE }\end{array}$ & $\begin{array}{l}\text { anti-diarrheal } 1^{13,15,16} \text {, astringent }{ }^{3,10,11,13,14,16}, \text { bronchitis }^{7,10,11,15,17,18}, \\
\text { depurative } 9,11,14, \text { expectorant } \\
\text { diseases } \\
\text { 7,9,10,13,11,14,16,16, }\end{array}$ \\
\hline $\begin{array}{l}\text { Aroeira/ rind } \\
\text { Schinus terebinthifolius Raddi; S. molle L. / } \\
\text { ANACARDIACEAE }\end{array}$ & 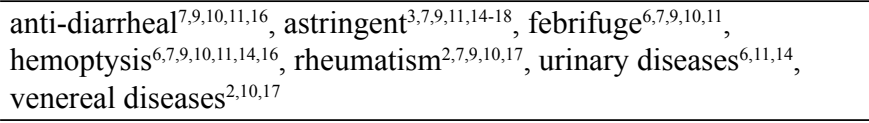 \\
\hline
\end{tabular}

Balsamo de copaíba/ balsam

Copaiba officinalis (L.) Kuntze/ C. coriacea (Mart.)

Kuntze/ C. langsdorfii (Desf.) Kuntze/ C. oblongifolia

(Mart.) Kuntze/ FABACEAE

Bálsamo de Tolu/ balsam

Myrospermum balsamum (L.) Harms/ FABACEAE

Barbasco/ leaves

Buddleja brasiliensis Jacq. Ex Spreng./ LOGANIACEAE

Barbatimão/ rind

Stryphnodendron adstringens (Mart.) Coville; $S$.

guyanensis Benth./ FABACEAE

Baunilha/ fruits

Vanilla planifolia Andrews/ ORCHIDACEAE

Cainca/ roots

Chiococca brachiata Ruiz \& Pav; C. anguifuga Mart./

Rubiaceae

Cajueiro/ rind

Anacardium occidentale L/ ANACARDIACEAE/

Calumba/ roots

Jatrorrhiza palmata (Lam.) Miers./ MenisPermaceAE

Cangerana/ rind

Cabralea cangerana Saldanha da Gama / MeliaceA

Camará/ leaves

Lantana camara L. / VERBENACEAE

anti-diarrheal $^{6,10,16}$, bronchitis ${ }^{6,10,12,14,16}$, leucorrhea ${ }^{5,6,12,17}$, cutaneous afections $^{5,6,13,16,17}$, skin wounds $s^{5,6,13,14}$, urinary diseases ${ }^{6,10,16}$, venereal diseases $^{2,5,6,12,14,17,18}$

asthma $^{6,10,11,13,15}$, balsamic ${ }^{7,10,11,13-15}$, bronchitis ${ }^{7,11,13,15}$, diuretic , $^{7,10,13}$, expectorant $^{10,13-15}$, respiratory diseases ${ }^{6,7,11}$, urinary diseases ${ }^{6,7,11,14,15}$ anti-hemorroidal ${ }^{2,6,10,15,16}$, arthritis ${ }^{6,10,15,16}$, bronchitis $^{5,6,10,11,15,16}$, emollient ${ }^{3,5,6,10,11,15-17}$, hemoptysis ${ }^{6,10,16}$, respiratory diseases ${ }^{5,6,10,11,15,16}$ anti-scorbutus $\mathrm{s}^{6,7,9,10,11,13,16}$, anti-diarrheal ${ }^{6,9,10,11,15-17}$,

astringent ${ }^{2,3,5,6,7,9,10,11,12}$, hemostatic ${ }^{6-16}$, leucorrhea ${ }^{6,7,9-17}$, metrorrhagia $^{6,11,15}$, skin wounds ${ }^{6,11,12,14,15,17}$, tonic ${ }^{7,10,11,15}$

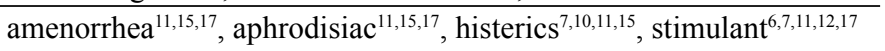

amenorrhea ${ }^{2,6,7,11-13,16}$, diuretic ${ }^{2,5-7,10-17}$, emetic $^{2,3,17}$, purgative ${ }^{3,5-7,11-19}$, rheumatism $^{10,13,14,19}$, snake bites $2,6,7,8,10-12,14,16,19$, cutaneous afections $\mathbf{s}^{6,7,10,15}$

astrigent $^{6,7,11,17}$, diabetes ${ }^{6,7,10-17}$, halitosis ${ }^{10,12,16}$, tonic $^{7,11,14,15}$ anti-diarrheal ${ }^{3,6,7,11,17}$, anorexia ${ }^{6,7,11}$, anti-emetic ${ }^{6,11,17}$, dyspepsia $^{6,7,11,17}$, stomach problems $s^{7,8,11}$, cutaneous afections $s^{6,7,15,17,18}$ tonic $^{3,11,17}$

emetic $^{3,6,17}$, purgative $e^{3,6,17}$ anti-tussive $\mathrm{e}^{8,10,13,15-18}$, asthma ${ }^{10,15-18}$, balsamic ${ }^{7,10,11,13,15}$, bronchitis $^{8,10,11,15,18}$, expectorant ${ }^{5,7,10,11,13,15}$, febrifuge $e^{7,10,11,13,14}$, rheumatism ${ }^{2,6,10-12,17}$, respiratory diseases ${ }^{2,6,11}$, cutaneous afections $^{6,11,12}$, tonic ${ }^{6,10,11}$, whooping cough ${ }^{10,11,15,16}$

Canela Sassafraz/ rind

Ocotea sassafras (Meisn.) Mez; Laurus sassafraz;

Sassafraz sassafraz/ LAURACEAE

Carapiá/ roots

Dorstenia multiformis var. arifolia (Lam.) Bureau; $D$. brasiliensis Lam.; D. reniformis Pohl/ Moraceae

depurative $e^{7,9,11,15,16}$, diaphoretic ${ }^{3,10,11}$, rheumatism ${ }^{4,6,7,10,11,13,15,16}$, sudorific $^{4,6,7,11,15,16}$, venereal diseases ${ }^{7,11,16}$ amenorrhea ${ }^{2,7,9,10,11,14,17}$, anemia ${ }^{7,11,15}$, anti-diarrheal ${ }^{2,6,7,11,19}$, chlorosis $4,5,6,7,15,17$, diuretic $\mathrm{c}^{2,3,6,11,12,14,19}$, diaphoretic $\mathrm{c}^{2,3,4,6,7,9,11,14}$, digestive disorders 1,2,5,6,7,10-12,15,17,19, emmenagogue em, $^{6,9,11-14,17,19}$, febrifuge ${ }^{2,5-7,9-13,15,17}$, gangrene ${ }^{6,7,12,17}$, snake bites ${ }^{3,4,7,8,9}$, stimulant $^{4,6,11,12,17}$, tonic $^{6,7,9,11,13,15,19}$ 
Name of Monography/ part

Species/ FamiLY

Carnaubeira/ roots

Copernicia cerifera (Arruda) Mart./ ARECACEAE

Caroba/ leaves

Jacaranda caroba (Vell.) A.DC; J. brasiliana Person./

BigNONIACEAE

Carqueja amarga/ whole plant

Asteraceae

Casca d'anta/ rind

Drymis winteri Winter/ WINTERACEAE

\section{Cassaú/ wood and roots}

Aristolochia cymbifera Mart. \& Zucc. \& variedades/

ARISTOLOCHIACEAE
Baccharis genisteloides Persoon; B. trimera (Less.) DC/

Uses in books of the 19th century until the 1970s

$\operatorname{arthritis}^{6,7,11}$, diuretic d,6,7,10,11,16 $^{\text {, rheumatism }}{ }^{4,6,7,10,11,16}$, cutaneous

afections $^{7,10,11,17}$, venereal diseases s,6,7,10,16 $^{4,10,14,15}$

rheumatism ${ }^{7,11,13,15,16}$, skin wounds ${ }^{6,10,14,15,18}$, cutaneous

afections $\mathrm{s}^{2,3,6,7,10,11,13,14,15-18}$, tonic ${ }^{6,11,14}$, urinary diseases ${ }^{7,10,11,13}$, venereal

diseases $3,6-8,10,11,14-18$

anemia $^{2,15,16}$, anorexia ${ }^{2,11,13,15}$, anti-diarrheal ${ }^{7,10,11,15,16}$, diabetes $^{6,15-19}$

dyspepsia $^{2,7,10,12,13,15,16}$, intermittent fevers $2,3,7,10,11,13,15,17$, liver

disorders ${ }^{6,7,10,13,16}$, stomach problems $s^{2,6,11,15,13,16,19}$, tonic i $^{2,3,6,7,10-13,17}$, vermifuge $e^{6,10,15}$

anemia $^{5,6,11,12,15,19}$, anorexia ${ }^{10,13,16}$, anti-emetic ${ }^{7,10,15,16,19}$, antiscorbutus

6,7,10,11-13,15, anti-diarrheal ${ }^{6,7,10,11,13,16}$, anti-spasmodic ${ }^{2,6,7,10,13,16,17,19}$,

convalescence $^{6,11,16}$, digestive disorders ${ }^{5,10,13,15,16}$, dyspepsias , $^{5-10-13,15,16}$,

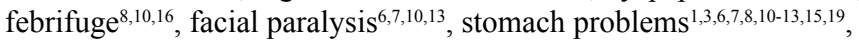
stimulant $^{1 \mathrm{a}, 2,12,12,17}$, tonic $\mathrm{c}^{1,1 \mathrm{a}, 2,5-7,111-13,15,17,19}$

Amenorrhea ${ }^{5,7,9,11,12,14,16,18}$, anti-diarrheal ${ }^{5,7,9,12}$, anti-convulsant ${ }^{5-7,11,16}$, antiseptic $^{2,7,10,16,17}$, anorexia ${ }^{2,9,10,14-17}$, chlorosis ch, $^{2,9,10,15-17}$,

dyspepsia ${ }^{4,5,7,10,15,16,19}$, diuretic ${ }^{2,4,6,7,11,14,19}$, diaphoretic ${ }^{2,6,7,10,15-17}$,

emmenagogue $2,7,9,11,14,17,19$, epilepsia ${ }^{7,11,14,16}$, intermittent

fevers $^{2,4,5-7,9,11-17,19}$, hysterics ${ }^{4,5,7,11,14,16}$, orchitis ${ }^{6,7,9,16}$, stimulant st, $^{2,9,14,15,17}$,

skin wounds $s^{4,5-7,9}$, snake bites s,4,6-9,11,14,17 stomach problems $s^{6,10,11,15,16}$, tonic ${ }^{2,7,11-13,15,19}$

Catuaba/ rizome

Anemopaegma mirandum (Cham.) Mart. ex DC/

BignONIACEAE/

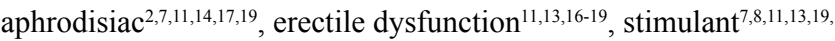
tonic ${ }^{7,11,14,13,16,19}$

Chá de pedestre/ leaves and flowers

Lippia pseudo-thea Schauer; Lantana pseudothea St.

Hil./ VerbenaceAE

Chapéu de couro/ leaves

Echinodorus macrophyllus (kunth) Micheli/

Alismataceae

Cipó azougue/ roots

Apodanthera smilacifolia Cogn./ CUCURBITACEAE

Cipó cabelludo/ whole plant

Mikania hirsutissima DC./ AsterACEAE

Cipó caboclo/ leaves

Davilla rugosa Poir./ DilleniaceAe

Cipó chumbo/ whole plant

Cuscuta umbellata Kunth/ Convolvulaceae

Cipó cravo/ wood

Tynnanthus fasciculatus Miers; T. elegantus Miers/

BignONIACEAE /

Cipó suma/ roots

Anchietea salutaris A. Saint-Hil./ VIOLACEAE

Coerana/ leaves

Cestrum laevigatum Schlecht./ SolanaceaE

Cordão de Frade/ whole plant

Leonotis nepetifolia (L.) R. Brown./ LAMIACEAE

Douradinha/ whole plant

Waltheria douradinha A. Saint-Hil./ STERCULIACEAE

Elemi/ balsam

Protium heptaphyllum (Aubl.) March.; P. icicariba

(DC.) March./ BurseraceaE

Espelina/ roots Cayaponia espelina (Silva Manso) Cogn./ CuCurbitaceae hemostatic ${ }^{6,10,13}$ expectorant $^{2,7,15}$, respiratory diseases ${ }^{2,6,7,810,15}$, rheumatism ${ }^{2,6,7,15}$, stimulant $4,6,7$

anti-inflammatory ${ }^{6,7,13}$, arthritis ${ }^{6,7,10}$, depurative $\mathrm{e}^{5,6,10,11,15}$ diuretic $^{5,7,8,11,13,15}$, urinary disorders ${ }^{6,10,15}$, hydrops $s^{7,8,10,13,15}$, liver disorders $^{6,11,15}$, rheumatism ${ }^{5,6,7,10,11,13,15}$, cutaneous afections $s^{4,6,7,10,11,13,15}$, venereal diseases $6,7,10$

Depurative $\mathrm{D}^{5,7,10,11,13,15}$, rheumatism ${ }^{10,11,15}$, cutaneous

afections $^{5,6,7,10,11,13,15}$, venereal diseases ${ }^{7,11,13,15}$

diuretic $^{7,10-13,15}$, urinary diseases ${ }^{1,6,7,10-13,15}$

astringent ${ }^{1,2,11,14,17,19}$, depurative $\mathrm{e}^{6,14,19}$, legs edema $\mathrm{a}^{1,1 \mathrm{a}, 10,11,15-17,19}$,

lymphedema ${ }^{7,11,15,16,19}$, orchitis $^{1 \mathrm{a}, 2,7,8,10,11,16,19}$

anti-diarrheal ${ }^{7,12,16}$, astringent ${ }^{6,11,12,16}$, diuretic ${ }^{7,12,16}$, hemoptysis ${ }^{3,6,7,10-}$

aphrodisiac ${ }^{6,15,19}$, carminative $\mathrm{e}^{7,11,13,19}$, dyspepsia ${ }^{10,11,15,19}$, stomach problems $^{7,10,11,13,15,19}$, stimulant ${ }^{6,10,13}$, tonic $^{7,11,15,19}$

depurative $e^{1,5-9,11,13,14,15}$, purgative $e^{1,5-7,10,17}$, cutaneous

afections ${ }^{1-3,6,7,9-11,13-15,17}$

anti-spasmodic $2,6,15,16$, emollient ${ }^{3,6,7,14,15}$, sedative e $^{3,6,7,14-16}$

asthma $^{6,7,10,15,16}$, anti-spasmodic $\mathrm{c}^{6,7,12,16}$, diuretic $\mathrm{c}^{6,7,10-12,14-16,18}$, metrorrhagia $^{12,13,15,16}$, rheumatism ${ }^{6,7,10-12,16}$, stimulant ${ }^{12,15,16}$, stomach problems ${ }^{13-15,18}$, tonic $\mathrm{c}^{7,11,14}$

anti-tussive ${ }^{1,3,8,15,17}$, bronchitis ${ }^{8,15,16}$, emollient ${ }^{1,1 \mathrm{a}, 3,8,17}$, respiratory diseases $^{2,6,10,15,16}$, skin wounds ${ }^{1,6,8}$, venereal diseases ${ }^{1 \mathrm{a}, 15-17}$

balsamic ${ }^{11,12,14}$

anti-diarrheal ${ }^{6,10,13}$, asthma $^{6,10,11,17}$, bronchitis ${ }^{6,10,11}$, dyspepsia $^{6,10,13}$,
${ }^{12,15,16}$, respiratory diseases ${ }^{6,7,10,11,15-17}$, skin wounds ${ }^{7,8,10-12,15,16}$, tonic $^{4,7,11}$ 


\begin{tabular}{|c|c|}
\hline $\begin{array}{l}\text { Name of Monography/ part } \\
\text { Species/ FAMILY }\end{array}$ & Uses in books of the 19th century until the 1970s \\
\hline Fedegoso/ roots & amenorrhea ${ }^{6,11,12,14,15}$, diuretic ${ }^{7,11,12,14,16,17}$, intermitent fever ${ }^{6,7,10-}$ \\
\hline Cassia occidentalis L.; Senna occidentalis / FABACEAE & $\begin{array}{l}12,15,16 \text {, hydrops }{ }^{7,10,12,15,16-18} \text {, liver disorders }{ }^{5,6,10,13,15-17}, \text { purgative }^{2,13,15,17} \text {, } \\
\text { tonic }^{5,7,11,12,14,17} \text {, vermifuge }\end{array}$ \\
\hline Gervão roxo/ leaves & debility $^{7,10,11,13,15}$, diuretic ${ }^{13,15,16}$, dyspepsia ${ }^{6,7,10,11,13,15,16}$, febrifuge ${ }^{2,7,10-}$ \\
\hline $\begin{array}{l}\text { Stachytarpha dichotoma (Ruiz \& Pav.) Vahl; } S \text {. } \\
\text { caienesnsis Cham; Verbena jamaicensis L./ VERBENACEAE }\end{array}$ & $\begin{array}{l}{ }^{12,14-16}, \text { hepatitis } \\
\text { sudorific }^{7,11,12,13,16}, \text { liver disorders } \\
\text { 11,13,15-17, }\end{array}$ \\
\hline \multicolumn{2}{|c|}{ Goiabeira/ rind } \\
\hline Psidium guayava Raddi./ MyrtaceAe & antidiarrheal $1^{6,7,10-12,17}$, astringent $6,7,11,12,14$ \\
\hline $\begin{array}{l}\text { Guaco/ leaves } \\
\text { Mikania glomerata Spreng; M. guaco Bonpl./ } \\
\text { AstERACEAE }\end{array}$ & $\begin{array}{l}\text { febrifuge }^{7,8,11,12,13,16}, \text { nevralgy } 1^{10,11,15} \text {, respiratory diseases } \\
\text { rheumatism }^{7,10-11,13,15,15,17} \text {, } \\
\text { diseases }^{7,11,16}\end{array}$ \\
\hline Guaraná/ seeds & anti-diarrheal ${ }^{6,7,11,12,13,16,17}$, depression ${ }^{7,11,13,15}$, migraine e,6,712,17, $^{2,7}$ \\
\hline Paullinia cupana Kunth; P. sorbilis Mart./ SAPINDACEAE & $\begin{array}{l}\text { nevralgy } y^{7,12,13,17} \text {, stimulant }{ }^{6,11,13,15} \text {, stomach problems }{ }^{2,6,7,11,13} \text {, } \\
\text { tonic }{ }^{7,8,11-13,15,17}\end{array}$ \\
\hline \multicolumn{2}{|l|}{ Guaycuru/ roots } \\
\hline Statice brasiliensis Boiss/ Plumbaginaceae & anti-diarrheal ${ }^{7,11,17}$, astringent ${ }^{7,11,17}$, hydrops $s^{7,11,17}$ \\
\hline Herva de bugre/ leaves & depurative $^{7,9,11,16}$, febrifuge $\mathrm{e}^{7,9,10,11}$, rheumatism ${ }^{7,10,11}$, cutaneous \\
\hline Casearia sylvestris Swartz./ FlaCOURTIACEAE & afections $s^{7,9-11,16}$, venereal disease ${ }^{7,9-11,16}$ \\
\hline \multicolumn{2}{|l|}{ Herva de Santa Maria/ leaves } \\
\hline Chenopodium ambrosioides L../ CHENOPODIACEAE & vermifuge $^{4-8,11,12,14,15,18}$, tonic $^{6,7,12,15}$, emmenagogue $^{6,7,8,12,15}$ \\
\hline Herva macahé/ leaves and flowers & anti-emetic $^{6,10,11}$, intermitent fever ${ }^{7,11,13,15}$, gastroenteritis ${ }^{7,10,15}$, \\
\hline Leonurus sibiricus L../ LAMIACEAE & rheumatism $^{8,11,13}$, stomach problems $s^{6,7,10,11,13,15}$ \\
\hline \multicolumn{2}{|l|}{ Herva de passarinho/ leaves } \\
\hline Struthanthus marginatus (Desr) Blume./ LoRANTACEAE & bronchitis $^{4,9,11-13,15}$, hemoptysis ${ }^{9,12,15}$, leucorrhea ${ }^{9,10,12,15}$ \\
\hline Herva tostão/ roots & diuretics $^{2,4,7,10,11,13,16,17,19}$, intermitent fevers ${ }^{6,11,13,15,19}$, hemoptysis ${ }^{10,15,16}$, \\
\hline Boerhavia hirsuta Jacq./ NyCTAGINACEAE & liver disorders ${ }^{2-4,7,8,10,11,13-17,19}$, urinary diseases ${ }^{4,7,11,16,19}$ \\
\hline Imbaúba/ shoots & anti-diarrheal ${ }^{2,6,11,12}$, asthma $^{6,10,11}$, cardiotonic $^{6,7,10-12,14,16}$ \\
\hline Cecropia hololeuca Miq.; C.pachystachya Trec.; & diuretic $^{2,6,9,12-14}$, leucorrhea ${ }^{2,6,9,12,13}$, respiratory diseasess ${ }^{6,7,9,10,11,13,16}$, \\
\hline C.palmata Willd./ CeCROPIACEAE & skin wounds ${ }^{2,6,8}$, venereal diseases ${ }^{2,9,12}$, whooping $\operatorname{cough}^{2,6,7,9,10,11,13,16}$ \\
\hline Ipecacuanha/ roots & 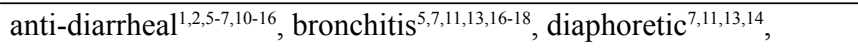 \\
\hline $\begin{array}{l}\text { Evea ipecacuanha (Brot.) Satandley; Cephaelis } \\
\text { ipecacuanha (Brot.) Stokes; C. acuminata Karsten/ }\end{array}$ & $\begin{array}{l}\text { emetic }^{1,2,4,5-8,10-17} \text {, expectorant } \\
\text { gough }^{1,6,2,5-12,17}\end{array}$ \\
\hline
\end{tabular}

RuBIACEAE

Jaborandi/ leaves

Pilocarpus jaborandi Holmes/ P. microphyllus Stapf ex $\quad$ bronchitis $^{7,10,11,13,16-18}$, diaphoretic din, 11,14,17 $^{\text {, diabetes }}{ }^{10,11,17}$,

Wardlewort/ RutACEAE

Jalapa do Brasil/ tubercule

Operculina macrocarpa (Linn) Urb./ CONVOLVUlaCEAE

Japecanga/ roots

Smilax japicanga Griseb. / SMILACACEAE

hydrops $^{7,10,11,13,17}$, sialagogue , $^{6,8,10,11,13,17,19}$, sudorific c $^{6,7,10,13,16,17,19}$

Jequitibá/ rind

Cariniana brasiliensis Casar./ LECYTHIDACEAE

Jurubeba/ roots

Solanum paniculatum L./ SOLANACEAE

Purgative $2,3,8,10,14,16,17$

anti-syphylitic $\mathrm{c}^{4,5,7,10,11,15-18}$, depurative $\mathrm{e}^{5,7,11,14,15,16}$, diuretic $\mathrm{c}^{4,5,7,11,13,14,15}$, gout $^{6,7,10,15,16}$, rheumatism ${ }^{4,6,7,10,11,13,15-18}$, cutaneous afections $\mathbf{s}^{2,4,6,7,9,11,13,15-17}$

Limoeiro bravo/ leaves

Siparuna apiosyce (Mart. Ex Tul.) A. DC./ MonimiaCEAE carminative $^{6,7,11}$, excitatory ${ }^{6,7,17}$

Mãe boa/ leaves

Cissus alata Jacq./ VITACEAE

astringent $t^{6,11,16}$, leucorrhea ${ }^{10,11,16}$, metrorrhagia ${ }^{10,11,16}$

anorexia $^{11,15,19}$, diuretic ${ }^{8,14,16,19}$ intermitent fevers $6,7,11,13,15,17,19$,

hepatitis $6,7,8,11,12,15,17$, jaundice ${ }^{6,7,8,11,13,15,16-19}$, liver disorders $6,7,8,11,16-19$, tonic $^{3,8,11,13-16,19}$

Mamoeira/ flowers

Carica papaya L./ CARICACEAE/

Manacá/ roots

Brunfelsia hopeana Benth.; Franciscea uniflora Pohl/

SolanACEAE

Maracujá/ leaves

Passiflora alata Curtis/ PAssifloraceAe

beriberi $^{6,7,13}$, rheumatism $^{6,7,10,11,13,16}$

bronchitis ${ }^{6,10,11,14}$, respiratory diseasess $s^{6,7,10,11,14}$

Depurative $e^{5,11,12,14}$, diuretic ${ }^{7,10,11,15,16}$, emmenagogue $e^{7,10,14,15,16}$, purgative $5,7,10,12,14,15,16$, rheumatism ${ }^{7,11,12,15,16}$, venereal diseases ${ }^{2,7,8,11,12,15-17}$

Convalescence ${ }^{5,10,12,17}$, sedative $^{5,7,9,11,13,19}$ 
Name of Monography/ part

Species/ FAMILY

Mate/ leaves

Ilex paraguariensis A. Saint-Hil./ AQUIFOLIACEAE

Matico/ leaves

Piper angustifolia Ruiz e Pavon/ PIPERACEAE

Milho/ stigma

Zea mays L./ POACEAE

Monésia/ rind

Chrysophyllum glycyphaelum Casaretto/ SAPOTACEAE

Muirapuama/ roots

Ptychopetalum olacoides Benth.; Liriosma ovata Miers/

Olacaceae

Mulungu/ rind

Erythrina mulungu Mart.ex Benth.; E. corallodendron

L./ FABACEAE

Nhandiroba/ seed

Fevillea trilobata L./ CuCURBITACEAE

Óleo de Sapucainha/ oil

Carpotroche brasiliensis (Raddi) Endl./ FlacourtiacEAE

Pacova/ seeds

Renealmia exaltata L.f.; Alpinia aromatica Aubl./

ZiNGIBERACEAE

\section{Paracary/ flowers}

Peltodon radicans Pohl/ Lamiaceae

\section{Pariparoba/ roots}

Heckeria umbellata (L.) Kunth; Pothomorphe

umbellatum/ Piper umbellatum H.B.K./ PIPERACEAE

Pau Pereira/ rind

Geissospermum laeve (Vell.) Miers; G. vellosi Allemão/

APOCYNACEAE

Quássia/ rind

Quassia amara L./ Picrasthma excelsa (Sw.) Planch./

SimARoubaceAe

Quina do campo/ rind

Strychnos pseudo-quina A.Saint-Hil./ LoganiaceaE

Quina mineira/ rind

Remijia ferruginea (A. St.-Hil.) DC./ RuBIACEAE

Ratanhia/ roots

Krameria argentea Mart. ex Spreng./ KRAMERIACEAE

Sabugueirinho do campo/ whole plant

Borreria centranthoides Cham. \& Schlecht./ RuBIACEAE

Sabugueiro/ flowers

Sambucus australis Cham. \& Schlech./ CAPrIfoliaceAE/

Salsaparrilha/ roots

Smilax papyracea Duhamel, S. syphilitica Griseb./

S. officinalis Kunth/ S.medica Schltdl. \& Cham./

Smilacaceae

Sapé/ rizome

Imperata exaltata Brong.; I. brasiliensis Trin/ POACEAE

Simaruba/ rind

Simaruba amara Aubl.; S. officinalis, D. C./

SimaroubaceAe

Sucupira/ rind

Bowdichia virgilioides Humbolt, Bonpland, Kunth; $B$. major/FABACEAE

Tayuiá/ roots

Cayaponia tayuya (Mart.) Cogn.; Trianospermum tayuial

Cucurbitaceae
Uses in books of the 19th century until the 1970s

convalescence $^{7,10,15}$, diuretic ${ }^{6,9,11}$, digestive disorders ${ }^{2,5,6,9,10,11,15}$, sudorific $^{2,9,17}$, stimulant ${ }^{2,6-11,15,17}$, tonic ${ }^{6,7,9,15}$

antidiarrheal ${ }^{4,7,11,12,17}$, astringent ${ }^{4,11,14,17}$, balsamic ${ }^{4,7,11,14}$, diuretic ${ }^{3,7,11}$, hemostatic $\mathrm{c}^{4,7,11,12,14,17}$, leucorrhea ${ }^{4,7,10,12,17}$, venereal diseases $3,4,7,10,11,12,17$

diuretic $^{2,7,10,11,13-15,17}$, urinary diseases ${ }^{2,6,7,10,11,13,15,17}$

anti-diarrheal $^{6,7,10,11,13,17}$, astringent ${ }^{2,3,6,7,10,11,17}$, hemostatic ${ }^{6,7,10,11}$, tonic $^{7,11,17}$

aphrodisiac $^{6,7,11,13,19}$, depression ${ }^{7,11,17,19}$, digestive disorders ${ }^{7,11,12,16}$, erectile dysfunction $^{7,11-13,16,19}$, facial paralysis ${ }^{11,12,16}$, rheumatism $^{6,7,11,19}$, tonic $7,12,13,16$

anti-tussive $\mathrm{e}^{6,7,15}$ asthma $^{7,11,15,17,19}$, bronchitis ${ }^{7,11,17}$, hepatitis ${ }^{6,10,15,16}$,

liver disorders ${ }^{7,10,11,15,16,19}$, sedative s-7,11-15,17,19 $^{5}$

liver disorders ${ }^{5,10,12,16}$, purgative $e^{5,10,12,16,17}$

morphea $^{8,9,15}$, cutaneous afections ${ }^{5,9,13,15}$, vermifuge $e^{6,12,13}$

stomach problems $\mathbf{s}^{6,10,11,15}$, vermifuge $e^{4,5,6,8,10,12,15,16}$

asthma $^{6,7,10,16,17}$, anti-tussive $e^{5,7,10,17}$, balsamic ${ }^{2,5,10,11}$, carminative $^{2,6,10,16}$, diuretics $^{2,6,10,16}$, snake/insect bites ${ }^{6,10,11,16,17}$, cutaneous afections $s^{7,10,11,16}$, venereal diseases ${ }^{6,7,10}$

diuretic $^{2,6,7,9,10,12,14,15,16}$, febrifuge $e^{6,12,16}$, jaundice ${ }^{6,12,16}$, liver and spleen disorders ${ }^{3,4,6,7,9,11-16,19}$, sudorific ${ }^{6,12,16,17}$, stomach problems $\mathbf{s}^{6,7,9,12,13,16,17,19}$

anorexia $a^{7,11,13,15}$, digestive disorders ${ }^{7,11,13,15}$, intermittent

fevers ${ }^{2,3,5,6,7,10-12,13,15,17}$, tonic $\mathrm{c}^{3,6,7,10,11,13,15}$

anti-diarrheal ${ }^{6,10,15}$, carminative c,10,15,16, dyspepsias $^{2,6,7,11,15-17}$,

febrifuge ${ }^{3,7,12,17}$, stomach problems $\mathrm{s}^{6,7,11,15,16}$, tonic $^{2,3,7,11,12,17}$, venereal diseases $^{10,15,16}$

intermittent fevers ${ }^{1,2,3,6,8,10,15,17}$ tonic $^{6,10,15}$

intermittent fevers ${ }^{1-3,10,13,14,15,17}$ tonic $^{6,8,11,10,13}$

anti-diarrheal ${ }^{6,10,11,14,15}$, astringent ${ }^{2,7,10,11,14,17}$

liver disorders ${ }^{7,11,13}$

diaphoretic $^{2,6,10}$, excitatory ${ }^{6,10,15}$, influenza ${ }^{11,15,16}$, rheumatism $^{6,10,15}$, sudorific $6,11,14,15,17$

depurative ${ }^{11,12,15,16}$, diuretics ${ }^{2,6,10,13,16}$, gout ${ }^{6,10,11,17}$,

rheumatism 6 ,7,10,11,13,16,17, sudorific $^{11,16,17}$, skin wounds ${ }^{6,7,13}$, cutaneous afections $^{2,6,10-13,16,17}$, venereal diseases ${ }^{3,4,6,7,11,12,15-17}$

diuretic $^{6,7,11,13}$, urinary diseases ${ }^{7,11,13}$

anti-diarrheal ${ }^{2,7,10-13,15-17}$, intermitent fevers ${ }^{2,7,12,13,15,16-17}$, febrifuge $\mathrm{e}^{3,7,11}$, hemostatic $^{12,15,17}$, tonic ${ }^{2,3,7,11-13,15,17}$, vermifuge ve, $^{2,15-17}$

Depurative $^{5,6,11,14}$, rheumatism ${ }^{2,7,11,13}$, cutaneous afections ${ }^{11,13,17}$, tonic ${ }^{7,11,15,17}$, venereal diseases ${ }^{10-13}$

depurative $^{5,7,8,11,16}$, diuretic ${ }^{10,11,17,19}$, purgative $^{2,5,7,17}$, rheumatism $^{11,13,16-19}$, cutaneous afections $^{5,10,11,15-18}$, venereal diseases ${ }^{6,7,10,11,15,16,19}$ 


\begin{tabular}{|c|c|}
\hline $\begin{array}{l}\text { Name of Monography/ part } \\
\text { Species/ FAMILY }\end{array}$ & Uses in books of the 19th century until the $1970 \mathrm{~s}$ \\
\hline $\begin{array}{l}\text { Tinguaciba/ rind } \\
\text { Xanthoxylum tingoassuiba A. Saint-Hil/ } \\
\text { RUTACEAE }\end{array}$ & $\begin{array}{l}\text { anti-spasmodic }^{5-7,10,11,13,15,16}, \text { carminative }^{7,11,13,19}, \text { dyspepsia }^{10,11,13,15,16,19}, \\
\text { intermitent fevers }{ }^{2,7,16,17,19} \text {, febrifuge } e^{7,11,13,19}, \text { stomach problems } \\
6,8,11,13,15\end{array}$ \\
\hline $\begin{array}{l}\text { Trapoeiraba/ whole plant } \\
\text { Tradescantia diuretica Mart. /COMMELINACEAE }\end{array}$ & 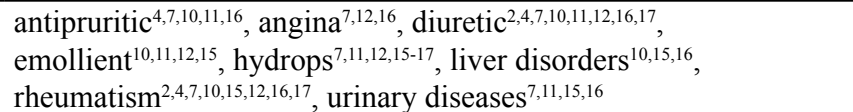 \\
\hline
\end{tabular}

by the first edition of the FBRAS, in 1929 (Guimarães, 2005). Although we have used the edition published in 1920 in this study, it should be noted that it describes the same data as the previous editions, published in $19^{\text {th }}$ century (Chernoviz, 1996).

\section{Ethnopharmacological survey on books of the $20^{\text {th }}$ century}

The data were extracted from fourteen books with data about plants collected before 1970 (Table 1). Five of these books were written by botanists who worked in different parts of Brazil. Included among these works was the Dicionário das Plantas Úteis do Brasil e das Exóticas Cultivadas (1926-1978), written by M. Pio Correia (1844-1934). Correia was a Portuguese author who had specialized in Economic Botany while working in Brazil, and who focused particularly on species with industrial potential. F.C. Hoehne (18821959) worked mainly in São Paulo and founded the Institute of Botany in 1942 (Franco and Drummond, 2005). His book Plantas e Substâncias Vegetais Tóxicas e Medicinais, first published in 1939, described the effects of several native plant species on animals. Another influential writer was J. Badini, a botany professor who worked at the Faculty of Pharmacy of Federal University of Ouro Preto for more than forty years (Badini, 1940). Also included in our study, was the French naturalist Paul Le Cointe, the first director of the Technical Chemical School of Pará. His book A Amazônia Brasileira was first published in French in 1922 (Le Cointe, 1947). Additionally, we utilized the writings of R. Braga, the first Director of the Agronomy School of Ceará, who wrote significant works including Plantas do Nordeste Especialmente do Ceará (Braga, 1960), as well as the work of Dias da Rocha published in 1997 (Matos, 1997). Four books used in this study were prepared by pharmaceutical laboratories. Among them were: Laboratorio Silva Araújo (Araujo \& Lucas, 1930), Laboratórios Catedral (Laboratórios Catedral, 1954) and Laboratório Rodomonte (Cruz, 1965). These books served as compilations in which they described data about the plants used in the preparations of their products. In addition, we used the work by Botsaris and Machado (1999), because it described the plants used in the preparation of the remedies from the Flora Medicinal Laboratory, which existed in Rio de Janeiro in the 1920s. The remaining books used were written by Monteiro da Silva (1911), Meira Penna (1941), Raul Coimbra (1942) and Balbach (1967). Data about pharmacological studies performed with the plants were obtained from Pubmed.

\section{Obtention and organization of the data}

We prioritized plants described in eightyseven Monographs from FBRAS, native to LatinAmerica that are found in Brazil. Species indigenous to America, but that do not grow here, such as guáiaco (Caribbean), boldo (Chile), quillaia (Panamá), quinas (Peru) or Peruvian balsam (Peru), were not included, since that the difficulties associated with obtaining the botanical material may difficult their use in local traditional medicine. Species without references about their origin were also not included, such as the Buchu (Manicaria saccifera), which was described as native to Amazonia only by Peckolt \& Peckolt. Data about each species were collected in the books by searching both scientific and popular names. The presence of any synonymies was checked at the site of the Missouri Botanical Garden (www.tropicos.org). Only data about the part of the plant (vegetal drug) described in the FBRAS were recorded. For example, while the leaves of the capeba (Pothomorphe umbellata) and herva tostão (Boerhavia hirsuta) are widely described in books as being medicinal, only data about the roots was included in Table 2. Data about the preparation of the remedies and doses were also not included. In Table 2, we have inserted only those medicinal uses that appear in three or more books ( $\geq 3$ books). This was necessary given the sheer volume and variety of traditional uses described for each plant in some bibliographies. Many uses that do not have any medical significance, such as "hydrophobia", "calmante do sangue," "inércia dos órgãos da geração," "resfriamento," "esurino," "descongestionante dos vasos genitais" or "asma úmida", for example, were not considered. We excluded other plants with traditional uses that are not medically relevant, including the amendoim (Arachis hypogaea) as aphrodisiac, araruta (Marantha arundinacea) that protects against insect bites, and borracha (Hevea brasiliensis), as an anthelmintic.

\section{RESULTS AND DISCUSSION}

Information on the use of native American 
species in Brazil was obtained from old bibliographies, written before 1970 (Table 1). It was important to limit our study to this time frame because after the 1950 s, Brazilian society experienced a series of intense transformations (Carvalho, 2003). These changes ranged from a reorganization of the Brazilian industrial park to a large cultural reordering that was characterized by intense repression of mysticism, including the traditional use of plants. These transformative processes were fully realized by the 1970 s, and they contributed to a significant loss of interest in medicinal botany (Brandão and Montemór, 2008; Manhã et al., 2008). This decline in popularity is clearly evident in the Brazilian Official Pharmacopoeia, in which most of the Monographs about medicinal plants were excluded from the second Edition, published in 1959 (Brandão et al., 2006; 2008a). Within the twenty books encompassed in this study, about 2,500 records exist that discuss the traditional uses for these pharmacologically relevant plants. The data refer to twenty-two roots, twentyone leaves, nineteen rinds, seven whole plants, seven flowers, three balsams, three seeds, two woods, one fruit, one oil, two rizome, one tubercule, one shoot and one stigma. Raul Coimbra is responsible for specifying the highest number of traditional uses for the plants, with 312 records. This was followed by Araujo and Lucas (295 records), M.P. Correia (245 records), Meira Penna (241 records), Cruz (232 records) and Balbachas (210 records). The other books with a high number of traditional indications for the plants were those of Chernoviz (170 records), Catedral (163 records) and Le Cointe (134 records). The numbers of uses recorded in the remaining books were 97 from Renato Braga's work, 96 from Martius, 77 from Botsaris and Machado, 63 from Monteiro da Silva, 58 from Hoehne, 55 from Badini, 44 from Caminhoá, 38 from Peckolt, 29 from Matos and 38 from Saint-Hilaire. Eighty-six different medicinal uses have been described by the authors examined here and those cited in three or more books are shown in Table 2. The most common of these were as diuretics and tonics, which accounted for twentyeight plant species. Anti-rheumatic and anti-diarrhetic indications were also very frequent among the useful species, corresponding to 22 and 21 plants, respectively. Nineteen plants were reported to treat venereal diseases, seventeen to heal cutaneous infections and thirteen to alleviate intermittent fevers and stomach problems. The other most frequently documented applications were treatments for bronchitis, as depurative and to treat liver disorders (12 species). Eleven species were cited as being useful as astringents, stimulants and febrifuges, or for the treatment of dyspepsia and urinary disorders. Properties involved in asthma therapy, and wound healing, as well as those that are purgative and sudorific are attributed to as many as eight species. Seven species were described as emmenagogues, vermifuges, and hemostatic agents. The other uses were indicated for one to six plant species.

The uses of some of species from Table 2 were already noted by both naturalists Saint-Hilaire and von Martius, already in the first decades of the $19^{\text {th }}$ century (Brandão et al., 2008b). The uses of Psychotria ipecacuanha as an anti-diarrhetic, emetic and expectorant, Strychnos pseudoquina and Remijia ferruginea as treatment for intermittent fevers, Dorstenia sp. as a therapy for liver disorders, Drymis winteri as a

Table 3. Example of traditional uses cited in the bibliography ( $\geq$ ten books/ 50\%) and evidences in recent pharmacological studies.

\begin{tabular}{lll}
\hline Uses/ total of plants & Plants (number of books) & Recent pharmacological studies \\
\hline Anti-diarrheal/ 21 & ipecacuanha (12) & Several studies (WHO 2007) \\
Diabetes/3 & cajueiro (10) & Alexander-Lindo et al., 2004; Ojewole, 2003 \\
Digestive disorders/ 6 & carapiá (11) & None \\
Diuretic/ 28 & cainca (12) & None \\
Emetic/ 3 & ipecacuanha (17) & Several studies (WHO 2007) \\
Expectorant/ 5 & ipecacuanha (13) & Several studies (WHO 2007) \\
Febrifugue/ 11 & abútua (12) & None \\
& carapiá (11) & None \\
Intermittent fever/ 13 & cassaú (14) & None \\
& pau pereira (11) & Bourdy et al., 2004. \\
Leucorrhoea/ 6 & barbatimão (11) & Ishida et al., 2006 \\
Liver disorders/12 & herva tostão (13) & None \\
& jurubeba (11) & None \\
Cutaneous afections/17 & pariparoba (12) & None \\
& cipo suma (12) & Di Stasi et al., 1999 \\
Tonic/ 28 & caroba (12) & None \\
Venereal diseases/ 19 & japecanga (10) & None \\
Vermifuge/ 7 & casca d'anta (11) & None \\
\hline & caroba (11) & None \\
& herva de santa maria (10) & Mac Donald et al., 2004 \\
\hline
\end{tabular}


tonic and Davilla rugosa as an astringent, for example, are detailed in their books about medicinal plants (SaintHilaire, 1824; von Martius, 1843). Many uses described by these naturalists were cited in the other books from $19^{\text {th }}$ century (Caminhoá, 1877; Peckolt \& Peckolt, 1887 1896) including: Chiococca brachiata and C. anguifuga as emetics, Dorstenia sp. and Tradescantia diuretica as diuretics, Jacaranda caroba, J. brasiliana, Anchietea salutaris and Smilax japicanga to treat cutaneous infections, Baccharis genistelloides and B. trimera as a cure for intermittent fever, Waltheria douradinha as an anti-tussive, and Boerhavia hirsuta to treat liver disorders. These descriptions dating back to the $19^{\text {th }}$ century serve as historical records to confirm that these species have a long tradition of medical applications.

The uses most frequently cited ( $\geq$ ten books) are described in Table 3, as well as data resulting from their corresponding pharmacological studies. Roots of Psychotria ipecacuanha (ipecacuanha) were used as emetics, antidiarrheal and expectorant while Boerhavia hirsuta (erva tostão) was utilized to treat liver disorders, as evidenced by the high frequency of references to these applications within 13 books. The use of $P$. ipecacuanha as an emetic, expectorant and anti-diarrhetic is well known and this species is considered an important medicine also by World Health Organization (WHO, 2007). Despite its wide use, no pharmacological studies have been performed with $B$. hirsuta, in order to verify its efficacy in treating liver disorders. Other examples of medicinal uses widely cited in the bibliography that have not been verified by pharmacological studies include the roots of Chiococca brachiata and $C$. anguifuga (cainca) as diuretics (12 books), D. wintery (casca danta) as a tonic (11 books), Smilax japecanga as a rheumatism treatment (10 books) and $J$. caroba (caroba) as a treatment for venereal disease (11 books). It is important to note that all studies performed with the species shown in Table 3 have confirmed their efficacy. Research has validated, for example, A. occidentalis (cajueiro) as a hypoglycemiant (Alexander-Lindo et al., 2004; Ojewole, 2003), G. laevae (pau Pereira) as an anti-malarial medication (Bourdy et al., 2004), $S$. adstringens (barbatimão) as treatment for leucorrhoea (Ishida et al., 2006), A. salutaris (cipó) as a remedy for cutaneous infections (Di Stasi et al., 1999) and $C$. ambrosioides (Santa Maria) as a vermifugal agent (Mac Donald et al., 2004).

For decades, Brazilian pharmaceutical companies have exploited the medicinal properties of numerous native species to create commercial products on the basis of traditional formulas. Since 1995, however, Brazilian governmental agencies have required scientific studies to prove the efficacy and safety of any herbal medicine before the therapeutic claims can be accepted completely (Brasil, 1995; Brasil 2008). Thirty-seven species from Table 2 (42.5\%) were already used by companies in Minas Gerais for preparing their products, but few species still in use today due the lack of pharmacological studies with them (Brandão et al., 2009).

\section{CONCLUSION}

The results of this study show that the American plant species described in the first edition of the FBRAS have a long tradition of use that has been confirmed by historical records. Few pharmacological studies on these widely utilized species have performed, but all of them confirmed their efficacy as medicinal agents. These encouraging results indicate that the medicinal properties of these historically used, native species warrant further attention. We suggest that a strong debate take place amongst the scientific public and policy makers in an attempt to improve and facilitate the pharmacological study of them.

\section{ACKNOWLEDGEMENTS}

The authors thank CNPq for fellowships and FAPEMIG (PPM/2007) for a research grant.

\section{REFERENCES}

Alexander-Lindo RL, Morrison EY, Nair MG 2004. Hypoglycaemic effect of stigmast-4-en-3-one and its corresponding alcohol from the bark of Anacardium occidentale (cashew). Phytother Res 18: 403-407.

Araújo JES, Lucas V 1930. Catálogo de Extratos Fluidos. Silva Araújo \& Cia. LTDA: Rio de Janeiro.

Badini J 1940. Arquiclamídeas Medicinais de Ouro Preto. Livraria mineira: Ouro Preto.

Balbach A 1967. As plantas Curam Ed. Ed. A Edificação do Lar: São Paulo.

Botsaris AS, Machado PV 1999. Flora Medicinal: Memento Terapêutico. Laboratório Flora Medicinal J. Monteiro da Silva: Rio de Janeiro.

Bourdy G, Oporto P, Gimenez A, Daharo E 2004. A search for natural bioactive compounds in Bolívia through a multidisciplimary approach. Part VI. Evaluation of the antimalarial activity of plants used by IsoceñoGuaraní Indians. J Etnopharmacol 93: 269-277.

Braga R 1960. Plantas do Nordeste Especialmente do Ceará. Imprensa Oficial: Fortaleza, 540pp.

Brandão MGL, Cosenza GP, Moreira RA, Monte-Mór RLM 2006. Medicinal plants and other botanical products from Brazilian Official Pharmacopoeia. Rev Bras Farmacogn 16: 408-420.

Brandão MGL, Montemor RLM 2008. Sabedoria antiga em risco. Cienc Hoje 42: 77-79.

Brandão MGL, Zanetti NNS, Oliveira GRR, Goulart LO, MonteMór RL 2008a. Other medicinal plants and botanical products from the first edition of the Brazilian Official Pharmacopoeia. Rev Bras Farmacogn 18: 127-136.

Brandão MGL, Zanetti NNS, Oliveira P, Grael CFF, Santos APP, Monte-Mór RLM 2008b. Brazilian medicinal plants described by 19th century European naturalists and in the Official Pharmacopoeia J Ethnopharmacol 
120: 141-148.

Brandão MGL, Cosenza GP, Stanislau AM, Fernandes GW 2009. Influence of Brazilian herbal regulations on the use and conservation of native medicinal plants. Environ Monit Assess doi: 10.1007/s10661-00 9-0899-4

Brasil 1995. Ministério da Saúde. Agência Nacional de Vigilância sanitária (ANVISA). Portaria 06 de 31 de janeiro de 1995. Brasília: Diário Oficial da União.

Brasil 2008. Ministério da Saúde. Agência Nacional de Vigilância Sanitária (ANVISA) Instrução normativa $n^{\circ}$ 5 de 11 de dezembro de 2008. Lista de Medicamentos Fitoterápicos de Registro Simplificado. http//:e-legis. anvisa.gov.br/leisref/public/showact.php

Caminhoá JM 1877. Elementos de Botânica Geral e Médica. Typographia Nacional: Rio de Janeiro, 2 vols.

Carvalho ACD 2003. Feiticeiros, burlões e mistificadores: criminalidade e mudança das práticas populares de saúde em São Paulo, 1950 a 1980. São Paulo: Editora UNESP, 172p.

Carvalho ACB, Balbino EE, Maciela A, Perfeito JPS 2008. Situação do registro de medicamentos fitoterápicos no Brasil. Rev Bras Farmacogn 18: 314-319.

Chernoviz PLN 1920 [1996]. A Grande Farmacopéia Brasileira. Formulário e Guia Médico (19a Edição). Ed. Itatiaia, Belo Horizonte.

Coimbra R. 1942. Notas de Fitoterapia. Laboratório Clínico Silva Araújo: Rio de Janeiro. 288pp

Correa M 1984. Dicionário das plantas úteis do Brasil e das exóticas cultivadas (6 vols). Rio de Janeiro: IBDF.

Cruz GL 1965. Livro verde das plantas medicinais e industriais do Brasil. $1^{\text {a }}$ Edição. Belo Horizonte. 2 vols. 863pp.

Dean W 1995. A ferro e fogo. História da destruição da Mata Atlântica Brasileira. Cia das Letras: Rio de Janeiro.

Di Stasi LC, Gomes JC, Vilegas W 1999. Studies on anti-allergic constituentes in the leaves and stems of Anchietaea salutaris var martiana (Violaceae). Chem Pharm Bull 47: 890-893.

Franco JLA, Drummond JA 2005. Armando Magalhães Corrêa: gente e natureza de um sertão quase metropolitano. Hist Cienc Saude-Manguinhos 12: 1033-1059.

Guimarães MRC 2005. Chernoviz e os manuais de medicina popular do Império. Hist Cienc Saude-Manguinhos 12: $501-514$.

Hering H 1912. Necrológio do Dr.Theodoro Peckolt. Revista da Flora Medicinal 108-127.

Hoehne FC 1939 [1978]. Plantas e substâncias vegetais tóxicas e medicinais. DBE: São Paulo.

Ishida K, Mello JC, Cortez DA, Filho BP, Ueda-Nakamura T, Nakamura CV 2006. Influence of tannins from Stryphnodendron adstringens on growth and virulence factors of Candida albicans. J Antimicob Chemother 58: 942-949.

Laboratórios Catedral 1954. Dicionário de Sinônimos das Plantas Medicinais Brasileiras Industrializadas pelo Laboratório Vegetal Catedral. Catedral: São Paulo, 42pp.

Le Cointe P 1947. Amazônia Brasileira. Árvores e plantas úteis. Companhia Editora Nacional: São Paulo, Rio de Janeiro, Recife, Bahia, Pará, Porto Alegre. 506 pp

Mac Donald D, Van Crey K, Harrison P, Rangashari PK, Rosenfeld J, Warren C, Sorger G 2004. Ascaridol-less infusions of Chenopodium ambrosioides contain a nematocide that is not toxic to mammalian muscle. $J$ Ethnopharmacol 92: 215-221.

Manhã EM, Silva MC, Alves MGC, Brandão MGL 2008. Plant - a bibliographic database about medicinal plants. Rev Bras Farmacogn 18: 614-617.

Martius CFP 1843. Systema de materia medica vegetal Brasileira. Typografia Universal de Laemmert: Rio de Janeiro.

Matos FLA 1997. O Formulário fitoterápico do Professor Dias da Rocha. UFC Edições: Fortaleza.

Ojewole JA 2003. Laboratory evaluation of the hypoglycemic effect of Anacardium occidentale Linn (Anacardiaceae) stem-bark extracts in rats. Methods Find Experim Clin Pharmacol 25: 199-204.

Peckolt T, Peckolt G 1887-1896. Historia das plantas medicinais e úteis do Brazil. ( $1^{\circ}$ fascículo) Companhia Typographica do Brazil: Rio de Janeiro, 6vols.

Penna M 1941. Dicionário Brasileiro de plantas medicinais. Oficinas Gráficas de A Noite: Rio de Janeiro. 302.pp

Saint-Hilaire A 1824. Plantes usuelles des Brésiliens. Grimbert Libraire, Paris.

Saint-Hilaire A 1825-1852. Flora Brasiliae meridionalis. Paris: Berlin Bibliopan.

Shanley P, Rosa NA 2005. Conhecimento em erosão: um inventário etnobotânico na fronteira de exploração da Amazônia Oriental. Bol Mus Para Emílio Goeldi 1: 147.

Silva FRM 1911. Contribuição para o estudo da flora Brazileira. Typographia do Jornal do commercio: Rio de Janeiro, 159pp.

WHO 2007. World Health Organization. Selected Monographsfor Medicinal Plants. Geneva,vol. 3. 